\title{
Autism Symptoms in Attention-Deficit/Hyperactivity Disorder: A Familial Trait which Correlates with Conduct, Oppositional Defiant, Language and Motor Disorders
}

\author{
Aisling Mulligan · Richard J. L. Anney · Myra O’Regan · Wai Chen • \\ Louise Butler · Michael Fitzgerald · Jan Buitelaar · Hans-Christoph Steinhausen • \\ Aribert Rothenberger - Ruud Minderaa - Judith Nijmeijer · Pieter J. Hoekstra • \\ Robert D. Oades · Herbert Roeyers · Cathelijne Buschgens · Hanna Christiansen • \\ Barbara Franke · Isabel Gabriels · Catharina Hartman · Jonna Kuntsi · \\ Rafaela Marco · Sheera Meidad · Ueli C. Müller · Lamprini Psychogiou • \\ Nanda Rommelse - Margaret Thompson - Henrik Uebel · Tobias Banaschewski • \\ Richard Ebstein · Jacques Eisenberg · Iris Manor • Ana Miranda • \\ Fernando Mulas · Joseph Sergeant · Edmund Sonuga-Barke · Phil Asherson · \\ Stephen V. Faraone · Michael Gill \\ Published online: 19 September 2008 \\ (c) Springer Science+Business Media, LLC 2008
}

Erratum to: J Autism Dev Disord DOI 10.1007/s10803-008-0621-3

The following is a corrected version of Table 4:

The online version of the original article can be found under doi: 10.1007/s10803-008-0621-3.

A. Mulligan · R. J. L. Anney · M. O'Regan · L. Butler ·

M. Fitzgerald · M. Gill

Trinity College, Dublin, Ireland

A. Mulligan $(\bowtie)$

Department of Psychiatry, Trinity Centre for Health Sciences,

St. James's Hospital, Dublin 8, Ireland

e-mail: mulliga@tcd.ie

W. Chen · J. Kuntsi · P. Asherson

Institute of Psychiatry,

London, De Crespigny Park, London SE5 8AF, UK

J. Buitelaar - C. Buschgens - B. Franke

Radboud University, Nijmegen, The Netherlands

H.-C. Steinhausen · U. C. Müller

University of Zurich, Zurich, Switzerland

A. Rothenberger - H. Uebel - T. Banaschewski

University of Göttingen, Göttingen, Germany

R. Minderaa - J. Nijmeijer · P. J. Hoekstra - C. Hartman University of Groningen, Groningen, The Netherlands

R. D. Oades · H. Christiansen

University Clinic for Child and Adolescent Psychiatry, Essen,

Essen, Germany
H. Roeyers · I. Gabriels

Ghent University, Ghent, Belgium

R. Marco - A. Miranda

University of Valencia, Valencia, Spain

S. Meidad · R. Ebstein · I. Manor

Geha Mental Health Center, Petak Tikvah, Israel

L. Psychogiou - M. Thompson - E. Sonuga-Barke

University of Southampton, Southampton, UK

N. Rommelse $\cdot$ J. Sergeant

Vrije Universiteit, Amsterdam, The Netherlands

J. Eisenberg

S. Herzog Memorial Hospital, Jerusalem, Israel

F. Mulas

University Hospital La Fe, Valencia, Spain

S. V. Faraone

SUNY Upstate Medical University, Syracuse, NY, USA 
Table 4 Cluster analysis: SCQ responses for ADHD probands

\begin{tabular}{|c|c|c|c|c|c|c|}
\hline Size of the clusters & ADI & $\begin{array}{l}\text { Cluster } 1 \\
31 \%\end{array}$ & $\begin{array}{l}\text { Cluster } 2 \\
22.5 \%\end{array}$ & $\begin{array}{l}\text { Cluster } 3 \\
21 \%\end{array}$ & $\begin{array}{l}\text { Cluster } 4 \\
18.5 \%\end{array}$ & $\begin{array}{l}\text { Cluster } 5 \\
7 \%\end{array}$ \\
\hline Conversations & $\mathrm{C}$ & ---- & ---- & ---- & ---- & -- \\
\hline Stereotyped utterances & $\mathrm{C}$ & ---- & ++ & --- & --- & +++ \\
\hline Inappropriate questions & $\mathrm{C}$ & -- & +++ & -- & - & ++++ \\
\hline Pronoun reversal & $\mathrm{C}$ & ---- & --- & ---- & ---- & + \\
\hline Neologisms & $\mathrm{C}$ & ---- & + & ---- & ---- & ++ \\
\hline Verbal rituals & $\mathrm{R}$ & ---- & - & ---- & ---- & +++ \\
\hline Compulsions and rituals & $\mathrm{R}$ & ---- & - & ---- & --- & +++ \\
\hline Inapprop. facial expression & $\mathrm{S}$ & ---- & ---- & ---- & --- & -- \\
\hline Use of other's body to commun. & $\mathrm{S}$ & ---- & --- & ---- & ---- & - \\
\hline Unusual preoccupations & $\mathrm{R}$ & ---- & --- & ---- & ---- & - \\
\hline Repetitive use of objects & $\mathrm{R}$ & ---- & + & ---- & --- & ++++ \\
\hline Circumscribed interests & $\mathrm{R}$ & ---- & + & ---- & --- & ++ \\
\hline Unusual sensory interests & $\mathrm{R}$ & ---- & -- & ---- & ---- & - \\
\hline Hand and finger mannerisms & $\mathrm{R}$ & ---- & -- & ---- & ---- & -- \\
\hline Complex body mannerisms & $\mathrm{R}$ & ---- & - & ---- & ---- & +++ \\
\hline Self injury & - & ---- & -- & ---- & --- & + \\
\hline Unusual attachment to objects & $\mathrm{R}$ & ---- & ---- & ---- & ---- & -- \\
\hline Friends & $\mathrm{S}$ & --- & --- & -- & - & + \\
\hline $4-5$ social chat & $\mathrm{C}$ & ---- & --- & - & + & + \\
\hline 4-5 imitation & $\mathrm{C}$ & ---- & ---- & -- & + & - \\
\hline $4-5$ point to express interest & $\mathrm{C}$ & ---- & ---- & --- & -- & --- \\
\hline 4-5 gestures & $\mathrm{C}$ & ---- & -- & ++++ & ++ & + \\
\hline $4-5$ nod yes & $\mathrm{C}$ & ---- & ---- & -- & -- & ---- \\
\hline 4-5 shake head no & $\mathrm{C}$ & ---- & ---- & -- & -- & ---- \\
\hline 4-5 eye gaze & $\mathrm{S}$ & ---- & -- & --- & + & ++++ \\
\hline 4-5 social smile & $\mathrm{S}$ & ---- & ---- & ---- & -- & + \\
\hline $4-5$ showing and directing attention & $\mathrm{S}$ & ---- & ---- & ---- & ---- & --- \\
\hline $4-5$ offer to share & $\mathrm{S}$ & ---- & ---- & ---- & - & ++ \\
\hline 4-5 shared enjoyment & $\mathrm{S}$ & ---- & ---- & ---- & --- & - \\
\hline $4-5$ offer comfort & $\mathrm{S}$ & ---- & ---- & ---- & -- & -- \\
\hline $4-5$ quality of social overtures & $\mathrm{S}$ & ---- & ---- & -- & --- & --- \\
\hline $4-5$ range facial expression & $\mathrm{S}$ & ---- & ---- & ---- & ---- & --- \\
\hline 4-5 imitative social play & $\mathrm{C}$ & ---- & ---- & ---- & +++ & ++ \\
\hline $4-5$ imaginative play & $\mathrm{C}$ & ---- & ---- & ---- & + & + \\
\hline 4-5 interested other children & $\mathrm{S}$ & ---- & ---- & ---- & ++ & +++ \\
\hline $4-5$ respond to other children & $\mathrm{S}$ & ---- & ---- & ---- & - & ++ \\
\hline $4-5$ attention to voice & - & ---- & -- & ---- & + & ++++ \\
\hline 4-5 imaginative play peers & $\mathrm{S}$ & ---- & --- & --- & +++ & ++++ \\
\hline $4-5$ group play & $\mathrm{S}$ & ---- & -- & ---- & +++ & +++ \\
\hline
\end{tabular}

Key: +'s indicate presence of symptom; -'s indicate absence of symptoms; ++++ indicates symptom present in $80-100 \%$; +++ indicates symptom present in 70-80\%; ++ indicates symptom present in 60-70\%; + indicates symptom present in 50-60\%; ---- indicates symptom absent in $80-100 \%$; - - indicates symptom absent in 70-80\%; -- indicates symptom absent in 60-70\%; - indicates symptom absent in 50$60 \%$

ADI domains: $\mathrm{S}=$ social reciprocal interaction domain, $\mathrm{C}=$ communication domain, $\mathrm{R}=$ repetitive behaviour and stereotyped patterns domain 